\title{
ENDANGERED CITY
}

GLOBAL INSECURITIES

A series edited by Catherine Besteman and Daniel M. Goldstein 
This page intentionally left blank 


\section{AUSTIN ZEIDERMAN}

\section{ENDANGERED CITY \\ The Politics of Security and Risk in Bogotá}

DUKE UNIVERSITY PRESS

Durham and London 2016 
(C) 2016 Duke University Press

All rights reserved

Printed in the United States of America on acid-free

paper $\infty$

Typeset in Minion Pro by Westchester Publishing Services

Library of Congress Cataloging-in-Publication Data

Names: Zeiderman, Austin, [date] author.

Title: Endangered city : the politics of security and risk in

Bogotá / Austin Zeiderman

Other titles: Global insecurities.

Description: Durham : Duke University Press, 2016. |

Series: Global insecurities |

Includes bibliographical references and index.

Identifiers: LCCN 2015045616

ISBN 9780822361435 (hardcover : alk. paper)

ISBN 9780822361626 (pbk. : alk. paper)

ISBN 9780822374183 (e-book)

Subjects: LCSH: Emergency management-Government policyColombia-Bogotá. | Natural disasters-Colombia-BogotáPlanning. | Risk management-Colombia-Bogotá. | Urban policy-Colombia-Bogotá.

Classification: LCC HV551.5.C7 Z453 2016 |

DDC $363.34 / 80986148-\mathrm{dc} 23$

LC record available at http://lccn.loc.gov/2015045616

Cover Art: Diego Delgadillo / Stockimo / Alamy 